\title{
Prevalencia de los diferentes trastornos motores primarios del esófago. Estudio prospectivo de 5.440 casos
}

\author{
Ana Henríquez $D^{2,3 a}$, Attila Csendes J ${ }^{1,3}$, \\ G uillermo Rencoret $\mathbf{P}^{1}$, Italo Braghetto $\mathbf{M}^{1}$. \\ Primary esophageal motor \\ disturbances among patients with \\ esophageal symptoms
}

Background: The esophagus can suffer several motor disturbances of striated or smooth muscle. Aim: To determine the presence of primary motor disturbances of the esophagus among a group of patients with esophageal symptoms. Material and methods: Prospective study of 5,440 patients consulting for heartburn, chest pain or dysphagia, with primary esophageal motor disturbances, studied between 1994 and 2004. All were subjected to an esophageal manometry with eight perfused catheters connected to pressure transducers. Results: Nineteen percent of subjects had a normal esophageal manometry, $60 \%$ had unspecific motor disturbances usually associated to gastroesophageal reflux, 13\% had a nutcracker esophagus, $5 \%$ had diffuse esophageal spasm, $2 \%$ had achalasia and 0,3\% had an hypertensive sphincter. Conclusions: Primary esophageal motor disturbances are common among patients with esophageal symptoms. A manometry should be performed to these patients (Rev Méd Chile 2007; 135: - ).

(Key words: Esophageal manometry; Esophageal motility disorders; Gastroesophageal reflux)

Recibido el 30 de junio, 2006. Aceptado el 10 de abril, 2007.

${ }^{1}$ Departamento de Cirugía y ${ }^{2}$ Gastroenterología, Hospital Clínico de la Universidad de Chile. ${ }^{3}$ Centro Privado de Laboratorio Manométrico. Santiago, Chile aTecnólogo Médico

L a única función fisiológica del esófago es el transporte de la comida deglutida desde la faringe hacia el estómago. Para esta función, consta de musculatura estriada en su tercio superior y de musculatura lisa en los 2 tercios inferiores. Está delimitado por 2 esfínteres en sus

Correspondencia a: Attila Csendes, MD, FACS (Hon). Director Departamento de Cirugía, Hospital Clínico J.J. Aguirre. Santos Dumont 999, Santiago, Chile. Fono: 56-2-7774387. Fax: 56-2-7775043. E mail: acsendes@redclinicauchile.cl extremos y un complejo mecanismo neuromuscular controla su funcionamiento ${ }^{1}$. Por lo tanto, a nivel de la musculatura lisa del esófago, pueden ocurrir múltiples trastornos motores primarios 0 secundarios a otras patologías que también comprometen el esófago.

El objetivo del presente estudio fue determinar la prevalencia de cada uno de estos trastornos motores en un gran número de pacientes estudiados, en un centro de investigación clínica dedicado a este tema desde hace 35 años. 


\section{MateRIAL Y MÉTODO}

Pacientes estudiados. Este estudio prospectivo comenzó en agosto de 1994, en un centro privado dedicado a manometría esofágica y ano-rectal, donde se estableció un protocolo de estudio y evaluación de estos pacientes, con el objeto de conocer la prevalencia de todos los trastornos motores en nuestro medio, en un gran número de pacientes. Este protocolo incluyó la interrogación por presencia de síntomas de reflujo gastroesofágico (GE), disfagia, dolor torácico o síntomas esofágicos, asimismo los hallazgos endoscópicos, histológicos o radiológicos que tenía el paciente. Los criterios de inclusión comprendieron a pacientes con estas características y que incluyeron a 5.440 pacientes. Fueron excluidos 591 pacientes con reflujo gastroesofágico operados y evaluados antes y después de la cirugía 2,3 , a 86 pacientes con esclerodermia ${ }^{6-8}$, por tener trastornos motores secundarios de la musculatura lisa, al igual que 15 pacientes con diabetes que presentaban trastornos motores esofágicos. También se excluyeron 6 pacientes con miastenia gravis ${ }^{9}$ y 14 pacientes con trastornos de origen neurológico con disfagia, por compromiso de la musculatura estriada del esófago cervical.

Estudio manométrico. Este procedimiento fue realizado en todos los pacientes, después de $8 \mathrm{~h}$ de ayuno y una leve anestesia faríngea ${ }^{10,11}$. El paciente se coloca en decúbito lateral izquierdo, habiendo introducido previamente, en posición sentada, un catéter de 8 canales de polivinilo por la boca. Este catéter tiene 4 sensores radiales con orificios laterales de $2 \mathrm{~mm}$ y 4 sensores separados a $5 \mathrm{~cm}$ de distancia entre sí (Synectics Medtronic, Estocolmo, Suecia). Este catéter es perfundido constantemente a un flujo de $0,2 \mathrm{ml} / \mathrm{min}$, y está conectado a transductores de presión dentro de un sistema computacional, que analiza todas las características motoras del esófago a través de un software especialmente diseñado para este propósito (Medtronic Dallas, Texas). Se determinó la presión del esfínter esofágico inferior (en $\mathrm{mmHg}$ ), el largo total y el largo abdominal (en $\mathrm{mm}$ ), la amplitud de contracción de la mitad distal del esófago torácico (en $\mathrm{mmHg}$ ), la duración (en segundos) y el porcentaje de peristalsis después de la deglución de $5 \mathrm{ml}$ de agua tibia ${ }^{4}$. Los mismos parámetros se evaluaron en la mitad proximal del esófago torácico. También se midió la presión de reposo del esfínter cricofaríngeo y de las ondas faríngeas (en $\mathrm{mmHg}$ ). Todos estos estudios fueron realizados por una tecnóloga médica con 28 años de experiencia dedicada a este laboratorio.

Definiciones. Para la presente investigación se definieron claramente los parámetros a evaluar y las características manométricas de sujetos normales, ya publicados en extenso previamente ${ }^{2,10-14}$.

a. Sujetos normales: presión de reposo del esfínter esofágico promedio de $18 \mathrm{~mm}$, con rangos de 12 a $25 \mathrm{mmHg}$. El largo total promedio es de $38 \mathrm{~mm}$, con un largo abdominal promedio de $14 \mathrm{~mm}$. El porcentaje de relajación del esfínter es $95 \%$ a $100 \%$ y se ubica, en promedio, a $41 \mathrm{~cm}$ desde la arcada dentaria. En el cuerpo del esófago, la presión intraluminal de reposo es menor que la intragástrica (-4 $\mathrm{mmHg}$ ). Las ondas distales tienen una presión entre 50 a $100 \mathrm{mmHg}$, con una duración de 2 a $4 \mathrm{~s}$ y entre $80 \%$ y $100 \%$ de las ondas son peristálticas. A nivel de la mitad proximal del esófago, las características motoras son enteramente similares; amplitud de ondas entre $50 \mathrm{y}$ $100 \mathrm{mmHg}$, duración de 2 a 4 s y $80 \%$ a $100 \%$ de peristalsis. El esfínter cricofaríngeo tiene una presión de reposo entre 50 y $120 \mathrm{mmHg}$, con un largo de 2 a $4 \mathrm{~cm}$ y una relajación muy rápida de $100 \%$ con la deglución 2,10 .

b. Acalasia esofágica: se define por 4 características manométricas: esfínter esofágico inferior hipertensivo, sobre $26 \mathrm{mmHg}$, con un promedio de $40 \mathrm{mmHg}$ (aun cuando esta característica no siempre está presente); relajación esfinteriana incompleta, cercana a 50\% con la deglución, que es lo característico; aperistalsis total del esófago torácico, presión de reposo intraesofágica positiva mayor que la fúndica. El esfínter cricofaríngeo y la faringe son normales ${ }^{12,13}$.

c. Esófago en cascanueces: corresponde a un trastorno motor del esófago torácico, teniendo ondas peristálticas en $90 \%$ a $100 \%$ después de las degluciones, pero con una amplitud y duración mayor que lo normal, con un mínimo de $20 \%$ de las ondas con amplitud mayor a 180 mmHg. Puede tener un esfínter esofágico inferior normal o hipertensivo ${ }^{11,15-18}$. 
d. Espasmo difuso: corresponde a un trastorno de la musculatura del esófago torácico con al menos $20 \%$ de las ondas de contracción de amplitud normal o disminuida, simultáneas, no peristálticas, con duración prolongada y con 3 puntas, en vez de punta única o 2 puntas como en una onda de contracción normal. Puede tener esfínter inferior normal o hipertensivo ${ }^{14,19-21}$.

e. Esfínter esofágico inferior hipertensivo: corresponde a un trastorno aislado exclusivo del esfínter esofágico inferior, con una presión de reposo elevada, sobre $40 \mathrm{mmHg}$, pero con una relajación esfinteriana completa, de $100 \%$, en respuesta a una deglución ${ }^{14,22}$.

f. Trastornos motores inespecíficos, también llamados «ipocontracción»primaria o secundaria del esófago torácico, que corresponden a la presencia de una motilidad esofágica inefectiva, con mayor porcentaje de las ondas de contracción con amplitud baja, generalmente menor a $30 \mathrm{mmHg}$, habitualmente asociadas a reflujo GE crónico $2-4,16,23$.

\section{RESULTADOS}

La prevalencia de los diferentes trastornos motores primarios del esófago en 5.440 sujetos estudiados manométricamente en un periodo de 10 años, se muestra en la Tabla 1. Hallazgos completamente normales se encontraron en $19 \%$ de los sujetos estudiados. El trastorno motor primario más frecuente, corresponde a los trastornos motores inespecíficos del esófago torácico, habitualmente en pacientes con síntomas de reflujo gastroesofágico $(60,4 \%)$. El segundo trastorno motor más frecuente fue el esófago en cascanueces en 13,4\%. La acalasia del esófago, que es un trastorno primario muy emblemático, sólo correspondió en este estudio a $2,1 \%$ del total de trastornos motores esofágicos. El hallazgo más infrecuente y ocasional es el esfínter GE hipertensivo aislado.

En la Tabla 2 se detallan la distribución por edad y género de cada uno de los grupos. Se aprecia que la mayoría de los trastornos motores ocurre en el adulto joven bajo 50 años, excepto en

Tabla 1. Prevalencia de los diferentes trastornos motores primarios del esófago $n=5.440$

\begin{tabular}{|lrr|}
\hline Diagnóstico & \multicolumn{2}{c|}{$\mathrm{n}$} \\
\hline - Normales & 1.034 & $(19 \%)$ \\
- Trastornos primarios & 4.406 & $(81 \%)$ \\
a. trastorno inespecífico & 3.286 & $(60,4 \%)$ \\
b. cascanueces & 731 & $(13,4 \%)$ \\
c. espasmo difuso & 257 & $(4,7 \%)$ \\
d. acalasia & 117 & $(2,1 \%)$ \\
e. esfínter GE hipertensivo & 15 & $(0,3 \%)$ \\
\hline
\end{tabular}

GE =gastroesofágico

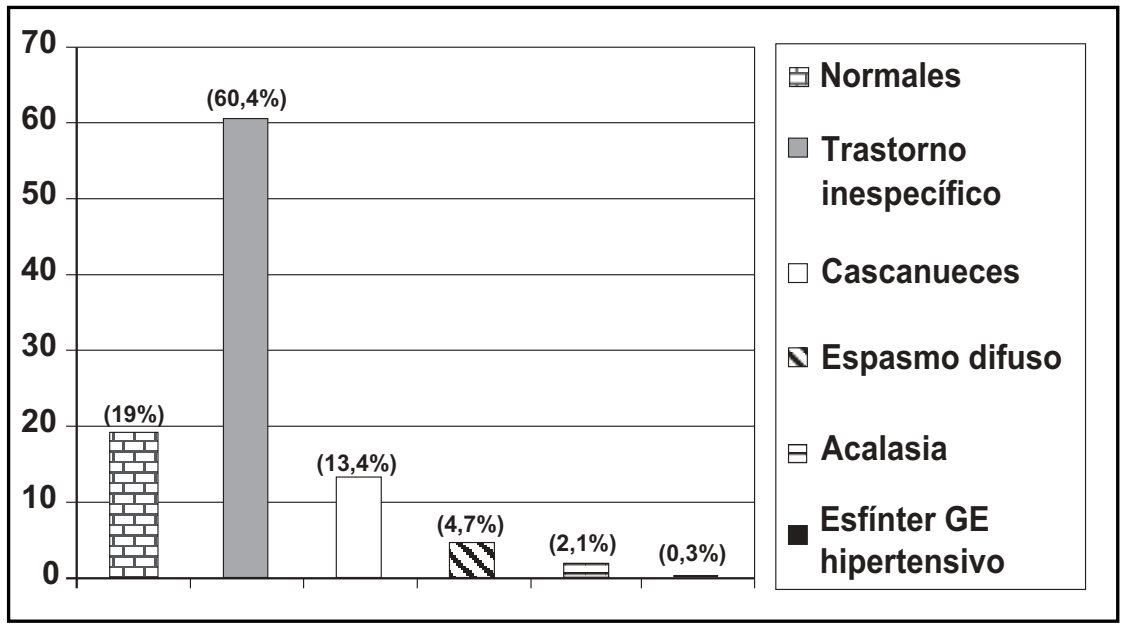

Figura 1. Prevalencia de los diferentes trastornos motores primarios del esófago. 
Tabla 2. D istribución por edad y sexo de los pacientes con diferentes trastomos motores primarios del esófago $\mathrm{n}=5.440$

\begin{tabular}{|lrrrr|}
\hline Diagnóstico & $\mathrm{n}$ & $\begin{array}{c}\text { Edad promedio } \\
\text { (años) }\end{array}$ & \multicolumn{1}{c|}{ Mujeres } & Hombre \\
\hline Normales & 1.034 & $44,1(10-73)$ & $624(60,3 \%)$ & $410(39,7 \%)$ \\
Trastornos & & & & \\
$\quad$ inespecíficos & 3.286 & $48,3(9-78)$ & $1.865(56,7 \%)$ & $1421(43,3 \%)$ \\
Cascanueces & 731 & $48,2(17-83)$ & $565(77,3 \%)$ & $166(22,7 \%)$ \\
Espasmo difuso & 257 & $54,4(19-77)$ & $172(67,3 \%)$ & $85(32,7 \%)$ \\
Acalasia & 117 & $40,8(8-73)$ & $61(51,8 \%)$ & $56(48,2 \%)$ \\
Esfínter & & & $12(80 \%)$ & $3(20 \%)$ \\
$\quad$ hipertensivo & 15 & $43,7(23-56)$ & & \\
\hline
\end{tabular}

el espasmo difuso, cuyos pacientes tienen una edad promedio de 54 años. La distribución por sexo en todos es muy similar, predominando las mujeres sobre los hombres, salvo en la acalasia, en que la proporción es enteramente similar.

\section{DisCUSIÓN}

El objetivo del presente estudio fue sólo analizar la prevalencia relativa de cada uno de los trastomos motores del esófago, en un gran número de sujetos estudiados mediante manometría computarizada en un periodo de 10 años, en un centro dedicado a estudios funcionales esofágicos, con una experiencia de 35 años. Es obvio que los resultados del presente estudio no se pueden extrapolar a lo que ocurre en la población general, sino sólo se pretendió definir su prevalencia local en un centro de estudios esofágicos. Tampoco es el objetivo del presente estudio analizar los síntomas predominantes en cada grupo de pacientes, que está ampliamente publicado en estudios previos $22,24,25$. Hace 15 años publicamos el primer estudio respecto a estas enfermedades en Chile ${ }^{14}$. En esa publicación, se evaluaron 1.250 pacientes estudiados en un periodo de 5 años, encontrando sólo 85 pacientes con trastornos motores primarios del esófago, que correspondió a 7\% del total similar a lo descrito por otro autor ${ }^{15}$. En ese análisis no se consideraron todos los trastornos motores inespecíficos secundarios o relacionados con el reflujo gastroesofágico y si los excluimos del presente estudio, los trastornos motores primarios corresponden a 20,5\%, 3 veces más que lo reportado previamente. Los motivos de este aumento pueden ser varios: consulta más frecuente por molestias esofágicas $\mathrm{y}$, por lo tanto, un mayor número de estudios esofágicos, aumento de interés de otros especialistas médicos como cardiólogos, broncopulmonares u otorrinolaringólogos para enviar pacientes que ellos atienden por dolor torácico o síntomas extraesofágicos para su estudio más precoz. También pudiera existir un real aumento de estas patologías, todas asociadas a un importante componente psíquico. Tal como se comentó previamente, la anatomía esofágica es única, ya que se compone de una musculatura estriada en la parte proximal y de musculatura lisa en los 2/3 distales, con 2 capas musculares: una interna o circular y una externa o longitudinal. Estudios recientes muestran que durante la peristalsis esofágica, un segmento de hasta $15 \mathrm{~cm}$, más que un punto focal del esófago, se contrae, permitiendo el desplazamiento de una onda con una curva en campana con un aumento único en la mitad de esta contracción ${ }^{26,27}$. La peristalsis iniciada por una deglución es una contracción secuencial de la musculatura a lo largo del esófago. Estudios actuales sugieren que hay una perfecta sincronía, en sujetos normales, entre la contracción peristáltica de ambas capas musculares ${ }^{27}$.

Los trastomos motores de la musculatura lisa del esófago se clasifican en primarios y secundarios ${ }^{14}$. En este estudio, sólo tratamos a los trastomos primarios, que son mucho más frecuentes que los trastornos secundarios. Tampoco nos hemos referido a los 
trastornos de la musculatura esquelética ${ }^{9}$, que se ven mucho en el área neurológica. No hay diferencias en género, tal como se ha descrito por otros autores ${ }^{24}$. Los síntomas más importantes de los pacientes, y por lo que fueron enviados a nuestro centro para el análisis manométrico, corresponden a disfagia, dolor torácico y pirosis. Cabe recordar que la disfagia en estos casos no es progresiva sino que es simultánea, tanto para líquidos como sólidos. La disfagia severa con compromiso metabólico es más característica de la acalasia que de los otros trastomos motores. Pacientes con trastornos motores inespecíficos con motilidad no efectiva, generalmente no presentan disfagia, sino más bien pirosis por vaciamiento esofágico lento y esofagitis. El dolor torácico se produce tanto por reflujo ácido como por un aumento de la sensibilidad a la distensión esofágica ${ }^{1,11}$. El estudio manométrico es el método de elección para evaluar los trastomos motores del esófago ${ }^{25,24,28}$. Se ha postulado que el estudio manométrico de $24 \mathrm{~h}$, que analiza la actividad motora con las comidas, es superior para el diagnóstico de los trastornos motores, comparado con la manometría tradicional ${ }^{28}$. Sin embargo, es un procedimiento poco práctico y que no lo tenemos en nuestro país. No hay duda que los trastornos inespecíficos son los más frecuentes, tal como lo muestra el presente estudio y otras publicaciones $^{25,17}$. Sin embargo, el más estudiado y que es el más frecuente de los trastomos motores primarios es el esófago en cascanueces ${ }^{23,25,14,15,17,20,27}$. El espasmo difuso es una entidad heterogénea ${ }^{19,21}$, difícil de diagnosticar claramente, ya que hay alteraciones estructurales, metabólicas o histológicas que pueden

\section{REFERENCIAS}

1. Mittal RK, BhaLa V. Oesophageal motor functions and its disorders. Gut 2004; 53: 1536-42.

2. Csendes A, Burdiles P, Maluenda F, Cortés C. Características clínicas y de laboratorio en pacientes con reflujo gastroesofágico crónico patológico. Rev Méd Chile 1998; 126: 769-80.

3. Csendes A, Maluenda F, Burdiles P, Henríquez A, Quesada S, Csendes P. Prospective study of esophageal motor abnormalities in patients with gastroesophageal reflux disease according to the severity of endoscopic esophagitis. Hepatogastroenterology 1996; 43: 394-9. similar este trastomo motor ${ }^{23}$. El antiguo concepto de que el espasmo difuso progresa hacia acalasia, en realidad ocurre raramente, en cerca de $8 \%$ de los casos $^{19}$.

No es el objetivo del presente estudio analizar el tratamiento de estos pacientes, que es variable según el diagnóstico que exista. Cuando hay evidencias de un reflujo ácido patológico, los inhibidores de la bomba de protones están indica$\operatorname{dos}^{11}$. Cuando hay dolor torácico intenso, otros derivados de nitratos o bloqueadores de la bomba de calcio pueden emplearse con éxito relativo ${ }^{1}$. Se ha descrito que el sildenafil es un potente relajador de la musculatura lisa y en un estudio reciente fue capaz de disminuir la amplitud de las contracciones esofágicas en $70 \%$, pero la mejoría clínica fue sólo de $40 \%$, con severos efectos colaterales ${ }^{30}$. Por lo tanto, otros tratamientos debieran ser usados en estos pacientes.

En resumen, los trastornos motores esofágicos son un hallazgo frecuente en pacientes que son enviados para un estudio manométrico por la presencia de dolor torácico, pirosis o disfagia, una vez descartada una causa orgánica como la responsable de estos síntomas. El tratamiento se guiará basado en los hallazgos manométricos del paciente.

\section{Agradecimientos}

Los autores agradecen a los 61 médicos que tuvieron la gentileza de enviarnos a los pacientes para su estudio manométrico y por enviamos los datos clínicos o de examen que faltaban para el completo análisis de sus pacientes.

4. Csendes A, Burdiles P, Alvarez F, Maluenda F, Henríuez A, Quesada S, Csendes P. Manometric features of mechanically defective lower esophageal sphincter in control subjects and in patients with different degrees of gastroesophageal reflux. Dis Esoph 1996; 9: 290-4.

5. Csendes A, Smok G, Quiroz J, Burdiles P, Rojas J, Castro C, Henriouez A. Clinical, endoscopic and functional studies in 408 patients with Barrett's esophagus compared to 174 cases of intestinal metaplasia of the cardia. Am J Gastroent 2002; 97: 554-60.

6. Miranda A, Csendes A, Guarda R, Aguilera S, Henríquez A, Ayala M. Alteraciones manométricas y radiológicas del esófago en pacientes con 
esclerosis sistémica progresiva. Rev Méd Chile 1979; 107: 730-2.

7. Velasco N, Olea E, González P, LuLo L, Csendes A. Cintigrafía esofágica en la evaluación de trastornos motores del esófago. Rev Méd Chile 1983; 111: 654-9.

7. Braghetto I, Csendes A, Urritia R, Cortés C, Prado A, Concha J. Características de la esofagitis severa en pacientes con colangenopatías. Rev Méd Chile 1992; 120: 1127-33.

8. Uribe P, Csendes A, LarRaín A. Compromiso esofágico de la esclerosis sistémica progresiva. Correlación clínica, radiológica y manométrica. Acta Gastroent Latinoam 1972; 4: 15-20.

9. Kuschel C, Csendes A, Guzmán S, Henríquez A. Motilidad esofágica en pacientes con miastenia gravis. Rev Méd Chile 1981; 109: 13-16.

10. Csendes A, Maluenda F, Braghetto I, Csendes P, HenRiquez A. Location of the lower oesophageal sphincter and the squamous columnar mucosal junction in 109 healthy controls and 778 patients with different degrees of endoscopic esophagitis. Gut 1993; 34: 21-7.

11. Csendes A, CÁrcamo C, Henríquez A. Características clínicas, endoscópicas, manométricas y magnitud del reflujo gastroesofágico en pacientes con esófago en cascanueces. Análisis prospectivo de 80 pacientes. Rev Méd Chile 2004; 132: 160-4.

12. Csendes A, URIBE P, LaRRaín A. Motility studies on fifty patients with achalasia of the esophagus. Am J Gastroent 1974; 62: 333-6.

13. Csendes A, Braghetto I, Henríquez A, Mascaró J. Late results of a prospective randomized study comparing forceful dilatation and esophagomyotomy in patients with achalasia. Gut 1989; 30: 299-301.

14. Csendes A, Díaz JC, Burdiles $\mathrm{P}$, Maluenda $\mathrm{F}$, Braghetto I, Henríquez A. Trastornos motores primarios del esófago no acalásico. Análisis manométrico. Rev Méd Chile 1989; 117: 285-92.

15. Pilhall M, Börjesson M, Rolny P, Mannheimer C. Diagnosis of nutcracker esophagus, segmental or diffuse hypertensive patterns, and clinical characteristics. Dig Disc and Science 2002; 47: 1381-8.

16. Mujica V, Mudipaw R, Rao S. Pathophysiology of chest pain in patients with nutcraker esophagus. The Am J Gastroent 2001; 96: 1371-7.

17. FANG J, BJoRKAm D. Nutcraker esophagus: GERD or an esophageal motility disorder. The Am J Gastroent 2002; 97: 1556-7.
18. Parrochia E. Esófago en cascanueces: una esofagopatía hipertensiva. Bol Hosp San Juan de Dios 2004; 51: 273-5.

19. Khatami SS, Khandwala F, Shay SS, Vaezi MF. Does diffuse esophageal spasm progress to achalasia? A prospective cohort study. Dig Dis Sci 2005; 50: 1605-10.

20. Tutuian R, Mainie I, Agrawal A, Gideon RM, Katz PO, CASTELL DO. Symptom and function heterogenicity among patients with distal esophageal spasm: studies using combined impedance-manometry. Am J Gastroent 2006; 101: 464-9.

21. Nguyen NQ, HoLoway RM. Recent developments in esophageal motor disorders. Curr Open Gastroent 2005; 21: 478-80.

22. Galmiche JP, Clouse RE, Balint A, Cook IJ, Kahrilas PJ, Paterson WG, Smout AJ. Functional esophageal disorders. Gastroenterology 2006; 130: 145965.

23. Clouse RE, Richter Je, Heading RG, Janssens J, WiLSON JA. Functional esophageal disorders. Gut 1999; 45: 1131-6.

24. Chang L, Toner BB, Fukudo S, Guthrie E, Locke GR, NoRTon NJ, SPECHER AD. Gender, age, society, culture and the patients prospective in the functional gastrointestinal disorders. Gastroenterology 2006; 130: 1435-46.

25. DiMarino AJ, Auen ML, Lynn RB, Zamani S. Clinical value of esophageal motility testing. Dig Dis Sci 1998; 16: 189-91.

26. Clouse RE, Staiano A, Bickston SJ. Characteristics of the propagating pressure wave in the esophagus. Dig Dis Sci 1996; 4: 2369-76.

27. Bhalia V, Padda B, Puckett J. Longitudinal and circular muscle contracts simultaneously in the esophagus during peristalsis. Gastroenterology 2004; 126: A-637.

28. Stein H, Singh S, DeMeester T. Efficacy of esophageal peristalsis: a manometric parameter of quantify esophageal body dysfunction. Diseases of the Esophagus 2004; 17: 297-303.

29. Jung HY, Puckett JL, Bhalia V, Rojas-Feria M, Bhargava V, Lu J, Mittal RK. Asynchrony between the circular and the longitudinal muscle contraction in patients with nutcracker esophagus. Gastroenterology 2005; 128: 1179-86.

30. ElVERER AJ, Schwetz I, HaNIMER HF. Effect of sildenafil on esophageal motor function in healthy subjects and patients with esophageal motor disorder. Gut 2002; 50: 758-64. 